\title{
A guide for open access
}

\author{
Along with five other journals in the Nature Portfolio, Nature Physics will pilot a new Guided Open Access trial \\ in 2021. We explain how it works.
}

W

hile the memories of 2020 will forever be associated with the individual and collective challenges we faced during the coronavirus pandemic, and as we look forward to the successful rollout of a vaccine programme that will hopefully bring about happier times, this year is also set to be one of consequential change for Nature Physics (and, for that matter, all Nature journals): we now offer authors the option of making their papers Open Access (OA).

As this development inevitably touches on the contrast between, on the one hand, the scientific ideal of free scholarly enquiry and the dissemination of discoveries and ideas, and on the other, the reality of the politics of research funding and the economics of publishing, it tends to stir strong emotions. Nature Physics is editorially independent of its publisher, Springer Nature, and our priority as a team of editors is to ensure that the content we publish is of the highest possible standard of quality and truth. Nevertheless, given the implications of the change to our publishing model, we believe it is appropriate to level with our authors and readers regarding how it works and why we are embracing it.

The article processing charge (APC) for authors wishing to make their manuscript OA after acceptance in Nature Physics is $€ 9,500$. Initially, we mostly expect authors that must comply with funder mandates - particularly those that are part of the cOAlition $\mathrm{S}$ consortium of funders - to take this option up. For most submitters, their papers will continue to be published under our traditional subscription model. However, as a so-called Transformative Journal, Nature Physics is committed to increasing the number of articles it publishes OA each year, with the eventual goal of becoming fully OA.

At this point, a natural question arises: what justifies such a cost? The simple answer is that a Nature article is expensive to produce. The work required to (among other things) knowledgably assess submissions, administer their peer review, develop the clarity of their exposition, achieve exacting standards of aesthetics in their artwork and production, as well as providing papers with a platform to achieve global impact requires the skill, time and experience of many people, which in turn require investment.

Historically, the cost of this work - and our contention is that it is valuable work has been spread over the many thousands of readers and institutions that subscribe to our journals. Making the articles free at the point of publication changes the equation, however, and flips this burden onto the authors, which are far fewer in number.

One way to reduce the cost for individual authors would be to drastically increase the volume of our output, by decreasing our selectivity. Many would argue that this would be a more inclusive approach, and there is no doubt that the institutional drive towards OA has shifted the publishing orthodoxy away from its bias to reject submissions. However, it is precisely because of the exponential growth of the scientific literature that we believe there remains a need for a venue that aims to highlight the most outstanding work the community has to offer.

So are there other ways to square this circle? We believe that there might be. Starting in January, Nature Physics is joining forces with Nature Genetics, Nature Methods, Nature Communications, Communications Biology and Communications Physics to trial a new publishing model called Guided Open Access.

The pilot offers Nature Physics authors the option of selecting to have their manuscript considered by three of the main physics venues in the Nature Portfolio (namely Nature Physics, Nature Communications and Communications Physics) with one single submission. The editors from each journal collaboratively assess the likely suitability of a manuscript for all three venues and, once the best fit is identified, assign responsibility of the file to the relevant editor who is entrusted with guiding the authors through the review process. Because the editorial criteria for these three journals span a much wider spectrum of possibilities than just Nature Physics alone, the chances of being sent out for review under Guided OA are correspondingly higher.

The handling editor will then arrange for the review of the paper and compile an Editorial Assessment Report, which contains an editorial evaluation, help to navigate and interpret the feedback from the reviewers, guidance for adhering to best-practice on openness, transparency and reproducibility, and a clear recommendation for a suitable outlet of publication, along with advice on the next steps required to help the authors achieve this goal. Unlike our standard submission route, there is an Editorial Assessment Charge of $€ 2,190$ for this service (which, to be clear, only applies for papers that are sent out for review). Authors that have their manuscripts eventually accepted in Nature Physics and Nature Communications will then be asked to pay a top-up APC of $€ 2,600$, whereas for those accepted in Communications Physics it will be $€ 800$.

By splitting the costs so that they reflect the different services that we provide, and by pooling aspects of them across some of our sibling titles, we are therefore able to provide a route to publish OA in Nature Physics for a total APC of $€ 4,790$. As part of the pilot, we will also enable authors to transfer their reports and the reviewers' identities (where they agree) to editorial offices at other publishers, and the papers that we do publish under the scheme will appear with the reviewers' reports alongside them. We do not expect this proposition to appeal to all authors - once again, we underline that Guided OA is an opt-in trial - but we hope that the combination of editorial efficiency, guidance and transparency it provides will be of interest to some.

Our decision to commit to OA is consistent with Nature's twofold mission (https://go.nature.com/2WhO3cw) of, first, serving scientists through the prompt publication of significant scientific advances, and second, ensuring that these results are rapidly disseminated to the public throughout the world, in a fashion that conveys their significance for knowledge, culture and daily life. This transition will take time, and we are only one player in a publishing ecosystem in which scientists, preprint servers, society journals and institutional funders will all play a role. We are determined to make it happen, while continuing to publish the most inspiring physics we can, wherever it may come from.

Published online: 6 January 2021 https://doi.org/10.1038/s41567-020-01151-6 